ISSN: 0213-2052 - eISSN: 2530-4100

DOI: https://doi.org/10.14201/shha202139163181

\title{
SISTEMAS DE DERIVACIÓN EN LA ANTROPONIMIA ANTIGUA DE LA PENÍNSULA IBÉRICA*
}

\section{Derivation systems in the ancient anthroponymy of the Iberian Peninsula}

José M. VALLEJO

Universidad del País Vasco (UPV/EHU)

josemaria.vallejo@ehu.eus

Fecha de recepción: 02-06-2020 Fecha de aceptación: 10-02-2021

ORCID: https://orcid.org/0000-0003-2266-1750

RESUMEN: El objetivo de este texto es subrayar la importancia de la onomástica personal en el estudio de las lenguas antiguas, tanto desde aspectos etimológicos como distribucionales. Aunque esto no es una novedad, porque las raíces antroponímicas han sido bien estudiadas, el artículo presenta la posibilidad del estudio de los sufijos mediante unos ejemplos demostrativos.

Palabras clave: antroponimia; onomástica; Hispania; áreas onomásticas; derivación.

ABSTRACT: The aim of this text is to underline the importance of personal onomastics in the study of ancient languages, from both etymological

* Este artículo se enmarca en el proyecto de investigación PID2019-106606GB-C32 del Ministerio de Ciencia e Innovación de España, y en el marco del Grupo Consolidado del Gobierno Vasco-Eusko Jaurlaritza GIC12/92-IT698-13. Tengo, además, una deuda de gratitud con los evaluadores externos y con la redacción de la revista, que han contribuido a mejorar el texto que el lector tiene ahora ante sus ojos. 


\section{JOSÉ M. VALLEJO \\ SISTEMAS DE DERIVACIÓN EN LA ANTROPONIMIA ANTIGUA DE LA PENÍNSULA IBÉRICA}

and distributional aspects. Although this is not a novelty, because the anthroponymic roots have been well studied, the article presents the possibility of the study of suffixes through some demonstrative examples.

Keywords: Anthroponymy; onomastics; Hispania; onomastic landscapes; derivation.

Al repasar la historia de la paleohispanística, queda fuera de toda duda que la onomástica se ha revelado como una herramienta útil en el estudio de las lenguas antiguas; no hay más que recordar la primera gran división lingüística de la península ibérica, efectuada con criterios toponímicos, que realizó Humboldt en 1821 al considerar los nombres en -briga como pertenecientes a una lengua diferente del ibero, concretamente al celta. Desde esta correcta intuición, la onomástica ha desempeñado un gran papel en el estudio de las lenguas peninsulares, especialmente en las regiones carentes de una epigrafía abundante. Esta ayuda que proporciona la onomástica, muchas veces entendida con cierto carácter intuitivo, debe ser fijada con criterios algo más científicos, lo cual, en una ciencia no exacta como la lingüística, es una labor difícil que necesita un gran consenso. En consecuencia, quienes evalúan la importancia de la onomástica (en especial, la toponimia) no logran un acuerdo total sobre su carácter exacto, dado que pueden considerarla:

- como un campo autónomo dentro de la lingüística (Nicolaisen 1990), que no necesitaría otros apoyos para ser explicado; téngase en cuenta la falta de referencia semántica en los nombres propios, los cuales, a diferencia del léxico común, no "significan», sino que simplemente «identifican». Así que, aunque se formen nuevos nombres, los mecanismos de derivación no coinciden totalmente con los de la lengua común. Por ello, la onomástica podría reflejar características o adaptaciones fonéticas o morfológicas que no se dan entre los apelativos. En ocasiones, el nombre es un indicador sociológico que, en el caso de la onomástica indígena antigua, puede ser estudiado en virtud de su constante asimilación a parámetros romanos;

- o bien como una disciplina auxiliar, que formaría parte de la lexicografía (Chambon y Hérilier 1993); al igual que ocurre en el léxico común, se pueden formar nuevas palabras o adquirirlas por préstamo, y tanto los nombres propios como los comunes comparten el rasgo de estar, en cierta medida, sujetos a modas. Y, si bien es cierto que, entre algunas culturas (como la comunidad semita), los nombres de persona son en cierto modo transparentes al estar mayoritariamente formados sobre 
raíces del léxico común, en gran parte del mundo lingüístico indoeuropeo la concepción de la onomástica es diferente; aun así, contamos con una gran tradición de autores que entendía la existencia de un nombre personal indoeuropeo originalmente compuesto y parlante ${ }^{1}$, cuya semántica recogía los valores de los elementos individuales. Este tipo de construcciones está presente en algunas de las lenguas indoeuropeas más tempranas, como el indo-iranio o el griego, cf. indio ant. Indradattas 'regalo de Indra', persa ant. Artaxerxes 'que domina con la

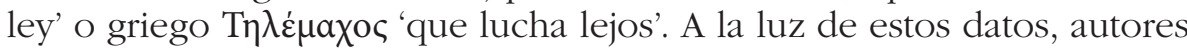
como H. Zimmer y K. Meyer extendieron la idea al irlandés y al resto de las lenguas celtas, cuya onomástica también respondía a un modelo claro de composición, al menos desde los primeros testimonios epigráficos continentales (galo Vercingetorix 'gran rey de los guerreros', irlandés ant. Cathmál 'príncipe de la lucha'). Evans (GPN 40-42) señalaba a este respecto una diferencia en el uso de la composición en celta (gala, galesa e irlandesa): mientras los compuestos eran corrientes entre los líderes o miembros de la aristocracia, las formaciones simples estaban reservadas a las clases más humildes. Una prueba de antigüedad podría ser su distribución geográfica dispar, lo cual quedaría también probado en lenguas cuya aparición en la historia es más tardía ${ }^{23}$. Pero esto conllevaría aceptar que las formas hipocorísticas (o simples), no desconocidas tampoco en estos sistemas lingüísticos, eran secundarias, esto es, que todo nombre simple provenía de un compuesto. Tal interpretación no era difícil de admitir para las lenguas donde abundaban los compuestos, como el indio, el celta, el griego y el germánico, pero el latín difería de estas en su predominio de los nombres simples. En estas circunstancias, Pulgram (1960) varió el punto de vista sobre el desarrollo del antropónimo indoeuropeo y se mostró partidario de que la forma originaria fuera el simple apelativo y no el compuesto.

También pueden mencionarse un par de características que, dentro de este grupo de coincidencias con el léxico, ha demostrado tener la onomástica. La primera es la capacidad de establecer diferentes áreas geográficas; el uso tanto de nombres comunes como propios puede fácilmente plasmarse en un mapa, y esto es revelador de cara a considerar, por ejemplo,

1. Pueden verse, a este respecto, obras clásicas como Bechtel (1917), Fick (1874) o Hilka (1910), entre otros.

2. En esta línea de tardía atestiguación se sitúan otras lenguas como el armenio y los grupos báltico, eslavo y germánico: cf. inglés ant. Eōmoer 'célebre por sus caballos', alemán ant. Hluderich 'rico en gloria', eslavo ant. Bogumils 'amado por Dios', etc. 
agrupaciones dialectales. La segunda es la posibilidad de emitir hipótesis sobre su etimología, es decir, sobre la lengua en la que esos nombres han sido formados, como el citado elemento - briga, cuya particular evolución fonética ${ }^{*} r_{0}>r i^{3}$ lo coloca entre las lenguas celtas.

Teniendo en cuenta estas consideraciones anteriores, y sopesando sus implicaciones reales, pensaríamos que, en cualquier caso, la onomástica tendría cierta relación con la lexicografía, aunque su posición exacta estaría más cercana de ser una disciplina tangencial antes que nuclear. Pero, a pesar de que este fuera un punto de vista válido en el estudio de la onomástica antigua, ello no tiene necesariamente que impedir nuestra ambición de buscar el origen etimológico de los nombres propios. Sin embargo, la mencionada falta de semántica de la onomástica va a ser un gran obstáculo para este objetivo.

Si nos centramos especialmente en la antroponimia (el estudio de los nombres de persona) nos percatamos inmediatamente de las dificultades. Veamos un ejemplo: como decíamos, un hispanohablante no suele ser consciente de la etimología de un antropónimo, ni siquiera de los más transparentes: alguien que se llame Vicente no se percatará de que su nombre significa 'el que vence' y que proviene del latín uincens, -ntis. Para acceder a su origen etimológico deberá, paradójicamente, «deconstruir» el nombre y volverlo a dotar de semántica (en cierta medida desgramaticalizándolo y privándolo de su marco referencial más habitual). Es decir, analizarlo supone volver a interpretarlo como si de un nombre común se tratara, lexicalizándolo de nuevo, de forma que recupere su semántica plena, con las dificultades que eso conlleva en las lenguas de atestiguación fragmentaria, sobre todo en aquellas que solo tienen una documentación onomástica. No obstante, el antropónimo no puede ser considerado - ni siquiera en este estadio - como un apelativo estándar, pues las formas expresivas que utiliza el hablante (Vicen, Vicentín, Chente $)^{4}$ no coinciden necesariamente con las que se utilizan para los apelativos. Esta forma de desandar el camino no es ni mucho menos intuitiva - sobre todo cuando desconocemos la lengua en la que fueron formados los nombres- y, como digo, debería estar más sujeta a criterios científicos.

3. La raíz indoeuropea * $b^{\text {h }}$ erg ${ }^{\text {h }}$ ' 'alto, elevado' (IEW 140-141, LIV 78-79) en grado cero deja una secuencia consonántica $\left({ }^{*} b^{\mathrm{h}} r g^{\mathrm{h}-}\right)$ en la que el elemento más audible, la $-r$-, desempeña el papel de núcleo de la sílaba. No todas las lenguas aceptaron esta secuencia, y algunas buscaron recursos para evitarla; las lenguas celtas son las únicas en el occidente europeo que desarrollan un apoyo con una vocal de timbre $-i$ - (> - ri-).

4. Y otras como Centico, Centito, Petete, Tente, Tete, Tico, Tintín, o formas más regionales como Visén o Bixintxo (cf., p. ej., Buesa 1988, 1634). 
Para poner en relación los dos puntos de vista anteriores podemos manejar como hipótesis que un nombre, que nace habitualmente en el marco de la lexicografía (sea como creación propia o como préstamo del léxico común o de otra lengua), a medida que se especializa como verdadero antropónimo, se separa de la semántica y adquiere incluso una manera autónoma de recibir expresividad por parte del hablante (aféresis, síncopas y apócopes, diminutivos, hipocorísticos o tal vez palatalizaciones, e incluso la forma en la que pueden establecerse los préstamos), que se suelen apartar del modo en que funcionan los apelativos en una lengua viva 5 .

Veamos un ejemplo antiguo de lo que estamos diciendo: un nombre hispano como Segontius (Mapa 1) puede analizarse en función de su pertenencia a la lengua común como derivado de una raíz que en su forma celta es *sego- 'vencer' (IEW 888-889; LIV 515-516; NIL 600-604) más el sufijo que corresponde al participio de presente activo *nt-, con lo que obtendríamos un resultado semántico similar a nuestro Vicente. No sabemos hasta qué punto el término pudo usarse como apelativo en la lengua de la región, o cuán ajeno resultaba a la semántica de la lengua mientras fue utilizado como antropónimo ${ }^{6}$.

Desde un punto de vista distribucional, puede analizarse en relación con el territorio en que se habló la lengua celtibérica, con cuya extensión geográfica viene a coincidir. La manera de construir las diferentes derivaciones (Segontius, Segontis, Segontieca...) nos puede hace pensar que seguramente era un recurso directamente relacionado con la lengua del portador más que con la lengua en la que estaba construido el propio nombre; así, para el hablante antiguo igual que para el moderno, a la hora de formar derivaciones del nombre, sería indiferente si se trataba de un nombre autóctono o de un préstamo. Hemos aprendido con la experiencia que la etimología desnuda no lo es todo en onomástica, sino que además hay otros procesos fonéticos y morfológicos que pueden caracterizar a un nombre propio. Formas como Etienne, Stephen o Esteban tienen la misma etimología, pero el diferente filtro fonético facilita a menudo su asignación a uno u otro sistema lingüístico. A pesar de una primera apariencia quizá pesimista, soy de los que creen que la onomástica tiene

5. La mera sonoridad de un nombre de persona puede ser suficiente para un préstamo en esta línea. Compárese la introducción en la década de los 90 del nombre Kevin en el caudal de antropónimos disponibles en nuestro país, a partir del nombre de un conocido actor de cine estadounidense.

6. De hecho, según los datos que pueden consultarse en $L I V$ 515-516 o en Matasović 2009,327 , no existen en las lenguas celtas formas verbales con esta raíz, con lo que su uso en la antroponimia bien pudo ser un residuo fósil. 
cosas que decir en los estudios diacrónicos de las lenguas y las sociedades, de una forma subsidiaria a la lingüística, y nunca al revés, por lo que no estaría justificado de ninguna manera el deseo de considerar a la lingüística como si fuera ella la auxiliar de la onomástica.

Concretamente en el campo de la antroponimia peninsular antigua, que tiene ya cierto recorrido y ha producido algunos frutos de interesante alcance, hemos orientado los estudios onomásticos básicamente desde dos ópticas que derivan de dos de las características mencionadas:

- la asignación de rasgos fonéticos en un nivel diacrónico, lo que lleva al establecimiento de etimologías;

- la geografía, que muestra la distribución areal de las formas.

1. Para ilustrar la primera, ya desde Humboldt sabemos que existen criterios fonéticos que orientan los análisis de algunos nombres claramente en una dirección lingüística: la presencia de formas con $r$ - inicial (como en el antropónimo Rectugenus) o con grupos consonánticos (como -br-, en los topónimos en -briga) le hicieron sospechar que seguramente no tenían una etimología ibera, porque tanto esta lengua como el vasco moderno carecen de estas características.

2. Y no hay más que ponerlos sobre un mapa para apreciar el alcance geográfico de su explicación etimológica. Tanto los nombres con $r$-inicial (Reburrus, Rectugenus...) como los topónimos con -briga se sitúan en la región occidental y septentrional de la península ibérica, en un área complementaria a la de las inscripciones ibéricas ${ }^{7}$. Otras características onomásticas como la presencia de aspiraciones, de grupos con sibilantes, de geminaciones o de $p$ - inicial también podemos caracterizarlas según sus rasgos y su distribución (Mapa 2).

A decir verdad, los mayores logros los hemos obtenido al conjugar con equilibrio estas dos potencialidades (la etimológica y la distribucional). Este tipo de trabajos deben ir siempre combinados para que resulten exponencialmente más ricos, y este artículo pretende, precisamente, ser un ejemplo de ello. Es verdad que no es la primera vez que esto se hace ${ }^{8}$, aunque los estudios antroponímicos se han basado sobre todo en la recopilación y sistematización de las raíces de los nombres, a partir de las cuales los pioneros como Untermann o Albertos pudieron definir unas áreas

7. Cf. los mapas correspondientes ya en Untermann 1961, mapa 4 (para -briga), y Rubio Alija 1959 (para Reburrus).

8. Una primera aproximación a estas cuestiones puede verse en Vallejo 2009. 
de concentración?. Gracias al avance informático y al desarrollo de grandes bases de datos, hoy día podemos manejar fácilmente todos los elementos y comprobar que el panorama no ha cambiado en gran medida, lo cual habla bastante a favor de aquellos primeros estudios ${ }^{10}$.

En efecto, un gráfico actualizado, resultante de la representación geográfica de las atestiguaciones onomásticas, confirma las conclusiones de aquellas obras pioneras; su éxito consiste en llegar a definir áreas lingüísticas coincidentes con las dibujadas por las atestiguaciones epigráficas directas de las diferentes lenguas y proporcionar información suplementaria sobre las lenguas antiguas.

Pero aparte de las elocuentes raíces onomásticas, hemos visto que existen otros fenómenos de la estructura onomástica que también pueden servir de ayuda para el estudio de los nombres: tanto los rasgos fonéticos como los procesos de derivación o composición son elementos muy útiles en el análisis de un sistema onomástico. Por citar algunos, en el campo de la fonética no podemos dejar de considerar procesos de sonorización, betacismo, existencia de aspiraciones, etc., que, en ocasiones, están geográficamente limitados y son fácilmente cartografiables. Incluso en el terreno de la composición nominal, ya se han hecho ciertos avances en otros ámbitos geográficos: Schmidt 1957 analizó los antropónimos compuestos de Galia, incluyendo básicamente toda la Keltiké; Raybould y Sims-Williams 2007a y 2007b estudiaron y cartografiaron los nombres compuestos de origen celta en Europa, con vistas a establecer su frecuencia por áreas. En la península ibérica, Untermann (1965, mapa 87) cartografió los nombres formados con el elemento -genos; y en Vallejo 2005b se recogieron todos los nombres compuestos del área indoeuropea de la península ibérica.

Uno de los ámbitos más fructíferos en cuanto a posibilidades de resultados lo constituye la derivación nominal, pues la abundancia de formas sufijales proporciona una riqueza que solo sería superada por la variedad de las raíces; lamentablemente, es este el dominio donde menos se ha incidido hasta ahora. El estudio quedaba básicamente limitado a los primeros trabajos de Untermann, que presentó en sus Elementos algunos mapas interesantes como el de las unidades suprafamiliares que contenían -oko- e -iko- ${ }^{11}$, o el sufijo -amo-, que parecía concentrarse preferen-

9. Trabajos importantes en esta línea fueron Untermann 1965 o Albertos 1983.

10. Cf. el Mapa 2 y su leyenda, comparado con la presencia general de la antroponimia indígena en Untermann 1965, 19.

11. El sufijo -ko- en formaciones secundarias en celtibérico recibió una atención especial por Rubio 2000. 
temente en las regiones atlántica y cantábrica ${ }^{12}$. Albertos utilizó también los mapas para representar la dispersión del sufijo -nd- (Albertos 1966, 286-287) y -nc- (Albertos 1966, 290-291). Así, teniendo en cuenta estos (pobres) antecedentes he intentado desarrollar un modelo de análisis de los componentes de derivación antroponímica (sufijos primarios o secundarios) buscando áreas de concentración y patrones repetidos que ayuden a complementar la información sobre las áreas onomásticas. El gran hándicap del estudio de sufijos frente al de raíces es que, mientras que estas se ordenan fácilmente por mera secuencia alfabética ${ }^{13}$, el estudio de los sufijos necesita de análisis previos, que requieren algo más de trabajo y de pericia.

Como he avanzado, la informática ayuda a sistematizar y a analizar mejor los nombres de acuerdo a nuestras pretensiones. Así, el trabajo de análisis y catalogación de cada uno de los sufijos ha sido bastante laborioso dado que la información no existía previamente, pero ahora disponemos por fin de información similar a la que ya manejábamos desde hace tiempo para las raíces; y, concretamente en el ámbito geográfico, la primera conclusión que extraemos es que hay varios tipos de sufijaciones: algunas se encuentran extendidas por áreas muy poco precisas, como la que dibujan las formas con el sufijo -ro-, presente en nombres importantes numéricamente como Caburus, Eburus, Pisirus o Reburrus. La explicación para esta dispersión geográfica amplia puede ser que se trata de un fondo de lenguas indoeuropeas, es decir, una familia lingüística única, por lo que tenemos que esperar un uso de algunos sufijos, en cierta medida, común. Una dispersión similar puede verse en la que dibujan otros formantes también muy usados en el ámbito indoeuropeo, como -ko-, donde también a sus variantes más numerosas (-oko-, -iko- o -ako-) les corresponde una distribución general hispana (cf. Mapa 3). En realidad, bastantes de los principales sufijos dejan una repartición geográfica familiarmente común, como -no-, -nd-, -lo-, -so- o -to-, todos ellos con bastante frecuencia de uso y con una distribución similar. Ciertamente no podemos decir que sean representativos de ninguna región en concreto, pero también ocurre lo mismo con algunas raíces onomásticas, como por ejemplo la de Ambatus (cf. mapas en Untermann 1965, 51-52 o Vallejo 2005, 135); a decir verdad, ello nos genera algunas dudas, dado que nos impide decidirnos si nos encontramos ante sufijos utilizados por la «misma

12. Cf. Untermann 1965, 197 y 192, respectivamente.

13. En la base Hesperia se puede obtener de un primer vistazo el elenco de nombres (aproximadamente 7000) y sus posibles agrupaciones en unas 2000 familias. 
lengua» (el "mismo sistema onomástico» en realidad) o por distintas lenguas (más o menos próximas) de una misma familia.

Por el contrario, otros sufijos presentan una mayor tendencia a documentarse preferentemente en algunas áreas concretas, como -eto- (cf. Mapa 4; algo menos claros son -ato- o -alo-), que muestran una gran predilección por la región occidental. Puede ser una buena excusa para la reflexión futura sobre diversas posibilidades de estudio, como la semántica de estos sufijos, a qué raíces se unen preferentemente, cómo se combinan entre sí, etc. En resumen, sería interesante saber si pueden asignarse con preferencia a la lengua de la región o son compartidos por más de un sistema lingüístico.

No faltan sufijos, aunque de menor frecuencia, con propensión a limitarse aún más geográficamente, de manera que podemos encontrarlos circunscritos a regiones más pequeñas en un mapa. De interés será saber si esta distribución más reducida con respecto al anterior grupo es debida a que son menos los ejemplos o que se trata de un único sistema lingüístico. Ejemplos representativos de una distribución más lusitano-galaica son aquellos nombres formados con los sufijos -amo- u -oso- (Mapa 5). Tampoco faltan otros casos con un centro de atestiguación astur, como -aiso- (Mapa 6), o celtibérico, como -aso- (Mapa 7).

A la vista de estos datos, una de las conclusiones que directamente podemos extraer es que los sufijos, tal y como nos han mostrado los gráficos, se comportan de manera similar a las raíces, es decir, tienen cierta capacidad de perfilar áreas lingüísticas, cumpliendo así una de las condiciones que definíamos anteriormente. Queda por ver si también presentan la capacidad de determinar etimologías ${ }^{14}$; esta constatación requerirá de cierto análisis combinatorio, verificando el tipo de raíces a las que se unen.

Una vez comprobado esto, el trabajo pendiente es grande: falta por saber a qué lengua(s) pertenecen todos estos sufijos, cómo se combinan entre sí, cuántos son primarios o secundarios, etc. Se me antoja que esta es una labor de ida y vuelta: inventariar y clasificar los sufijos nos ayudará a conocer el panorama lingüístico, y esto, a su vez, redundará en favor de establecer mejores etimologías y distribuciones geográficas.

El éxito de esta labor dependerá de nuestra habilidad a la hora de combinar las características y posibilidades de la onomástica con el material todavía en bruto que aquí hemos visto. Creo sinceramente que tenemos

14. Con toda seguridad lo hicieron, dado que sabemos que - $k o-$ o -yo- son sufijos derivativos muy usados en el ámbito indoeuropeo, similares en cierta medida a -no-; - eto- es una formación de agente en las lenguas celtas; -āto- se utiliza para expresar que el sujeto posee cierta cualidad (barbätus), -nt- es propio de participios de presente... 
asegurados algunos resultados que, en última instancia, nos ayudarán a conocer mejor los sistemas lingüísticos que se utilizaron en la península ibérica en la antigüedad.

Mapa 1. Segontius y derivados

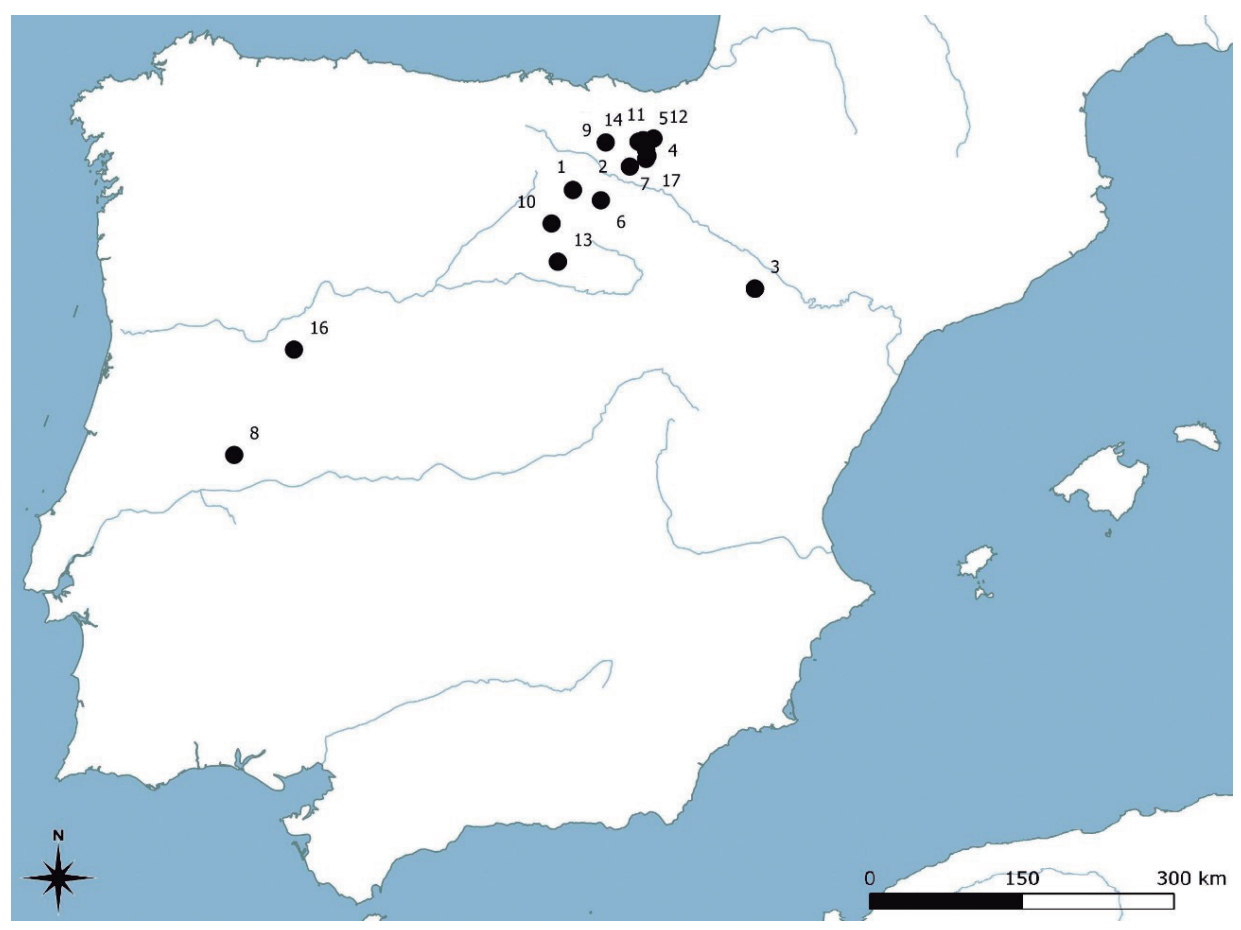

Hemos cartografiado:

1. Belorado (BU), Secontius, Secontia. 2. Bernedo (VI), [Sego/ntius. 3. Botorrita (Z), sekonzos, sekontios. 4. Contrasta (VI), Segontius. 5. Eguilaz (VI), [S]egontius. 6. Estollo (LO), Segontius. 7. Gastiáin (NA), Segontius. 8. Idanha-a-Velha (Idanha-a-Nova, CSB), Segontius. 9. Iruña (VI), [Sego] ntius. 10. Lara de los Infantes (BU), Secontius. 11. Ocáriz (VI), Segontiecus, Segontius. 12. Olazagutia (NA), Seg[ont]ieca. 13. Hallada en Oliva de Plasencia (CC). Segontius (Cluniensis). 14. Salvatierra (VI), Segontius. 15 = 13. Hallada en Três Minas (Vila Pouca de Aguiar VRE), Segontius (Cluniensis). 16. Yecla de Yeltes (SA). Segontius. 17. Zúñiga (NA), [Se]gontis?

Las referencias concretas pueden consultarse en Vallejo 2016 o en la Base onomástica del Banco de Datos Hesperia. 
MAPA 2. Áreas antroponímicas

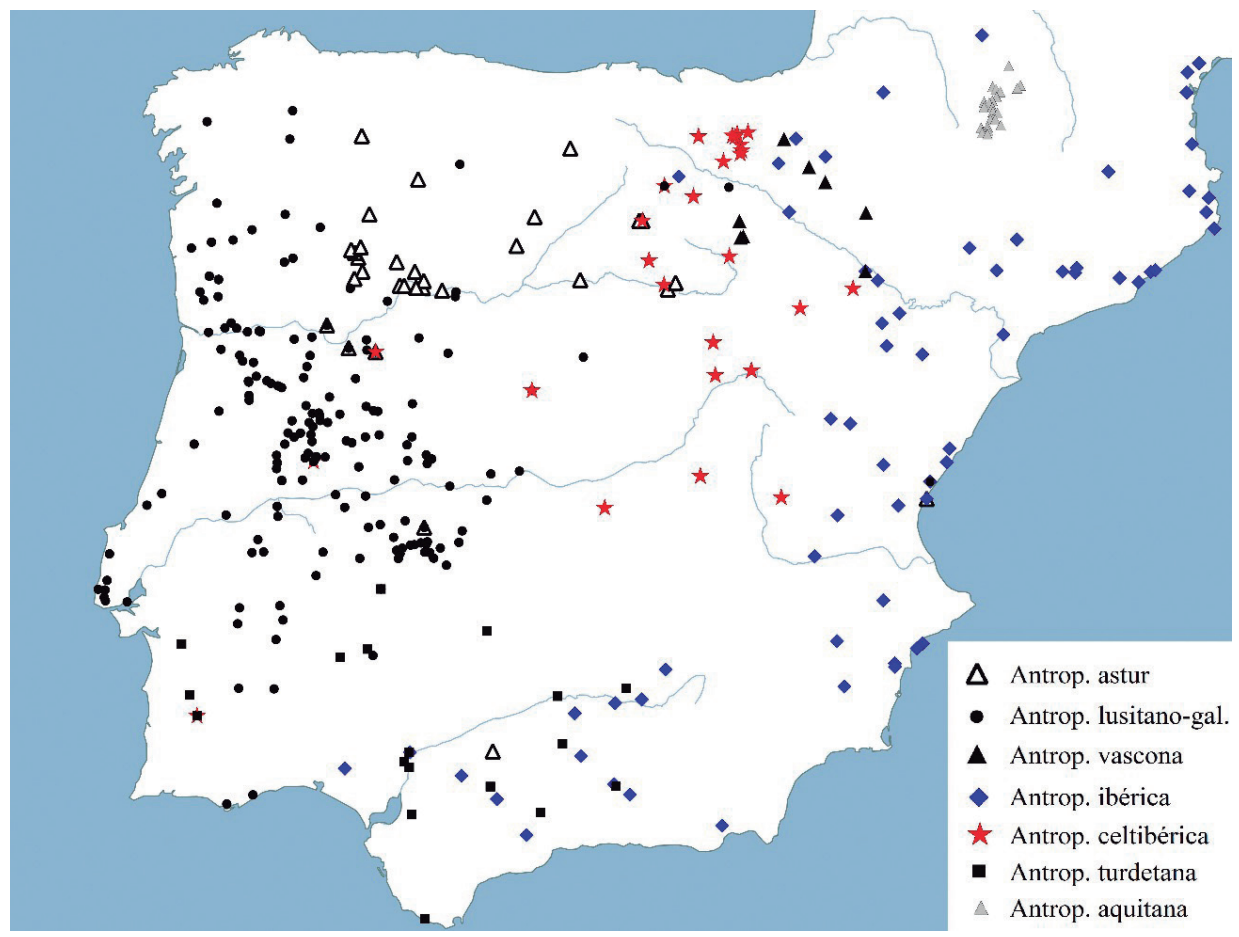

En este mapa, adaptado de Gorrochategui y Vallejo 2019, 347, están representadas algunas de las características más representativas de los antropónimos indígenas de la península. Como puede verse, se agrupan claramente formando áreas que pueden definirse por uno o varios de estos rasgos. El área celtibérica corresponde con gran exactitud al área de epigrafía celtibérica, con presencia de nombres personales como Segontius (cf. mapa 1), Rectugenus, Letondo o Melmandus (cf. Navarro, Gorrochategui y Vallejo 2011). La región ibérica, aun siendo menos densa, también tiene unos límites compartidos con la lengua ibérica; aquí están reflejados los elementos nominales más abundantes, como -adin, bilos-, il $(t) i$ - o tike-/tikers- (cf. Untermann, MLH III, § 7). La región vascona, con la presencia de los nombres con Abisun-, Sesen- o aspiraciones ausentes en otras regiones (Narbungesi, Serbuhoris o Vmmesahar, cf. Gorrochategui 1995). En la región turdetana se documentan nombres con Att- (como Attenius) o Sis- (Sisanna, Sisania, Sisean, Siseia, Sisbe; cf. Correa 2009; De Hoz 2010, 458). En el área lusitano-galaica, más abundante que el 
material epigráfico, tenemos nombres muy característicos como Tanginus, Camalus, Apana, Caturus o Sunua (cf. Vallejo 2005). En la astur se concentran nombres como Docius o Elaesus (cf. Vallejo 2013).

MAPA 3. -ko-

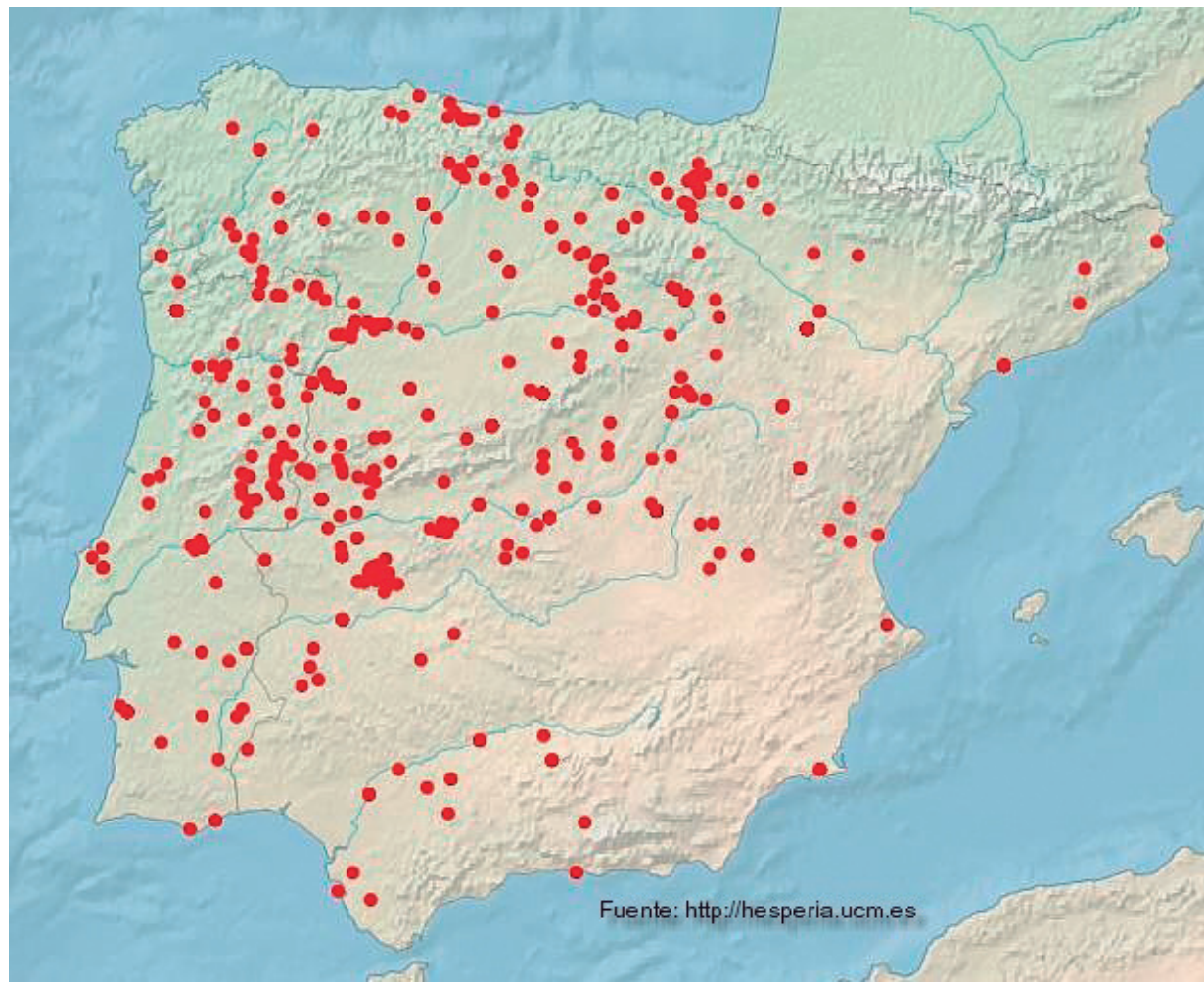

El mapa obtenido con las poblaciones en las que se documenta el formante - $k o-$ es el que se ve en la imagen, obtenido directamente sobre la Base de Datos Hesperia, donde pueden consultarse todas las referencias. Solo por citar los nombres que comienzan con $a$-, hemos cartografiado: Alticus, Ab(b)icus, Ablaidacoru(m), Ableca, Ablecaenus, Ableganga, Ablicus, Abliqum, abokum, Acceicum, Acinicus, Aebicus, Aelciocum, Aelcius, Aeturiq(um), Agolieca, Agrilicus, ailokiskum, ainolikum, Aiosecus, Ainica, Albeanca, Albic(i)us, -a, Albicianus, Albiganic(um), albinokum, Albucia, Alecius, Aleonicus, Alicia, alikum, alizokum, Allacarius, Alleicea, Allucius, Altica, Alticon, Aluccios, Aluc(c)ius, Amaca, Amaonicum, Amauca, Ambaicus, Ambatic(um), 
Ambaticorum, Ambatiq(um), Ambatus, Ambirodacus, Ameaicus, amikum, Ammaricum, Ammica, Ammicius, Ammonicus, -a, Ammonius, Amonicus, - $a$, Amusicus, Anecia, Aneq(um)?, Anica, Aniocum, Annicia, Annicum, Anniq(um), Annoca, Antilicus, Antuca, Apanicus, Apilicus, Apilocus, Aploniocum, Araca, Araica, araiokum, Arancia, Araniaq(um), Arauricus, -a, Arcaicum, Arceltius, Ardacus, arebasikos, Areiecus, Argamonica...

\section{MAPA 4. -eto-, -ato- y -alo-}

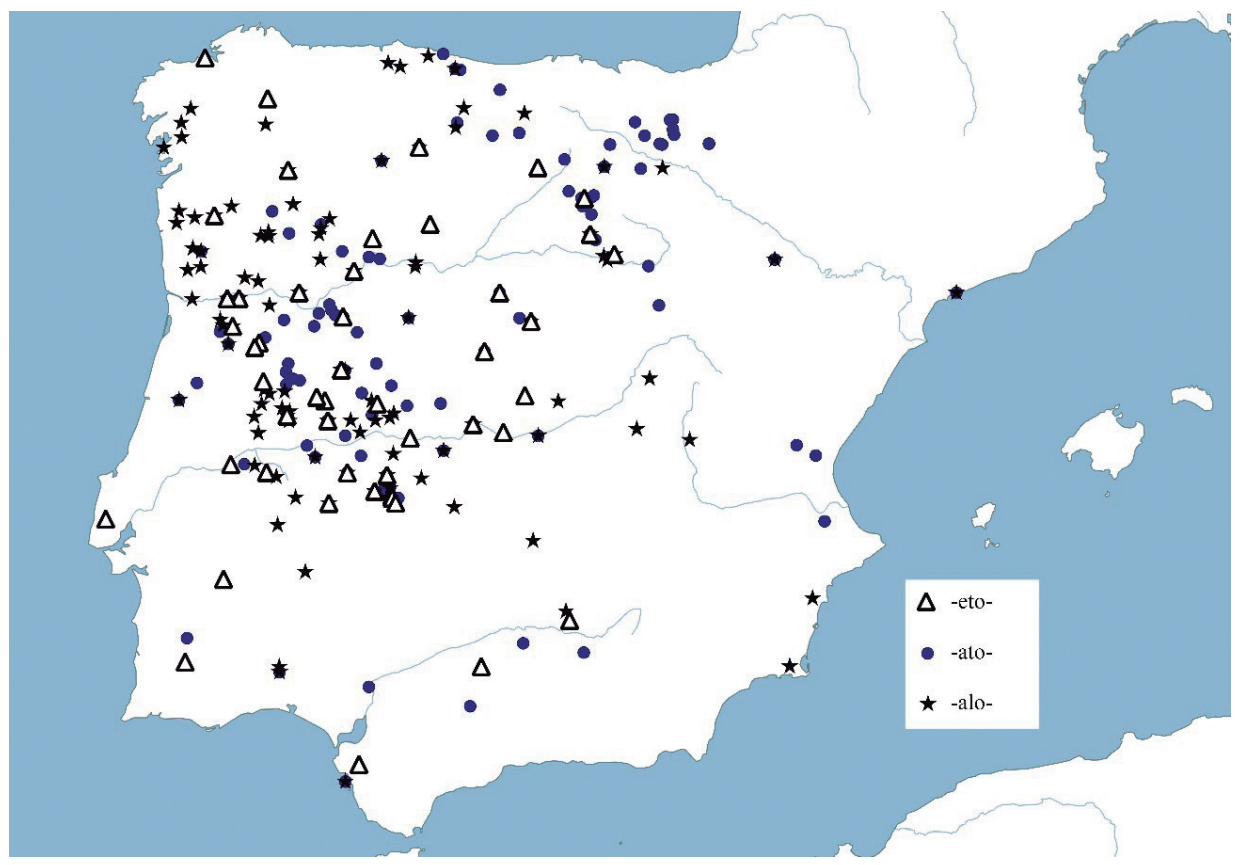

Los nombres que hemos cartografiado con el sufijo -eto- son:

Acces, Adaes, Aletius, Alles, Alpetus, Ance(i)tus, -a/Ange(i)tus, -a, Ancetolus, Annes, Annetia, Ares, Aretitonus, Atetus, Auettus, Caretosa, Caretus, Dancetus/Dangetus, Dobetianus, Dure(i)ta, Lomietus, Nemetius, Saletina, Seggues, Seguetus, Tarquetius, Toceta/Togeta, Tonceta/Tongeta, Toncetamus/Tongetamus, Tongeterus, Tongetus, Trigetus, Viannetiq(um), Vssuetio y Vxettius.

Con el sufijo -ato- tenemos:

Aegatia, Aelatius, Ambatus, -a, Ambatia, Ambatic(um)/Ambaticorum/ Ambatiq(um), Ambatio, Amitatis, Argatus, Assata, Assatus, balatioku, 
Balatuscun, Bouatis, Bouatus, Calatius, Celtiaticus, Celtiatis, Celtiatus, Culatus, Duatius, Durato, Ematia, kalatokum, Nitliata, Nusatita, Ouatus, Simatis, Tongatus, Treuoatus, ultatu, Vallata, Veiata, Ven(i)atus, Veratus, Viriatis y Viriatus.

Y con -alo- encontramos:

abaliu, abalos/Abalus, Acalla, Adalus, Apalus, Atalis, aualos/analoz, Aucaliq., Aucalus, Batalia, Battalus, Bercialliq(um), bibalos/Bibalus, Boualus, buntalos, Burdalicus, Cacala, Cacalia, Cacalo, Caebaliq(um)/ Caibaliq(um), Caelalio, Camalea, Camalicum, Camal(i)us, -a, Carcalia, Cebala, Comalius, Coralus, Cutalicus, Duentalius, Magalio, Munigalicus/Munigaligus, Necalica, Negalo, Negalus?, Otaliqum?, Samalo, Settal, Sogalus, Tabaliaenus, Tabalus, Tantalus, Tauitalis, Tirtaliqum, Tridallus, Tritalicus, uisalikum, urkala, Vbalacinus, Veibalus, Vendalo?, Visaligorum, Visal(i)us, -a, Vralus, Vrcala, Vrcalo, Vrcalocus.

Las referencias concretas pueden consultarse en Vallejo 2016 o en la Base onomástica del Banco de Datos Hesperia.

MAPA 5. -amo- y -OSO-

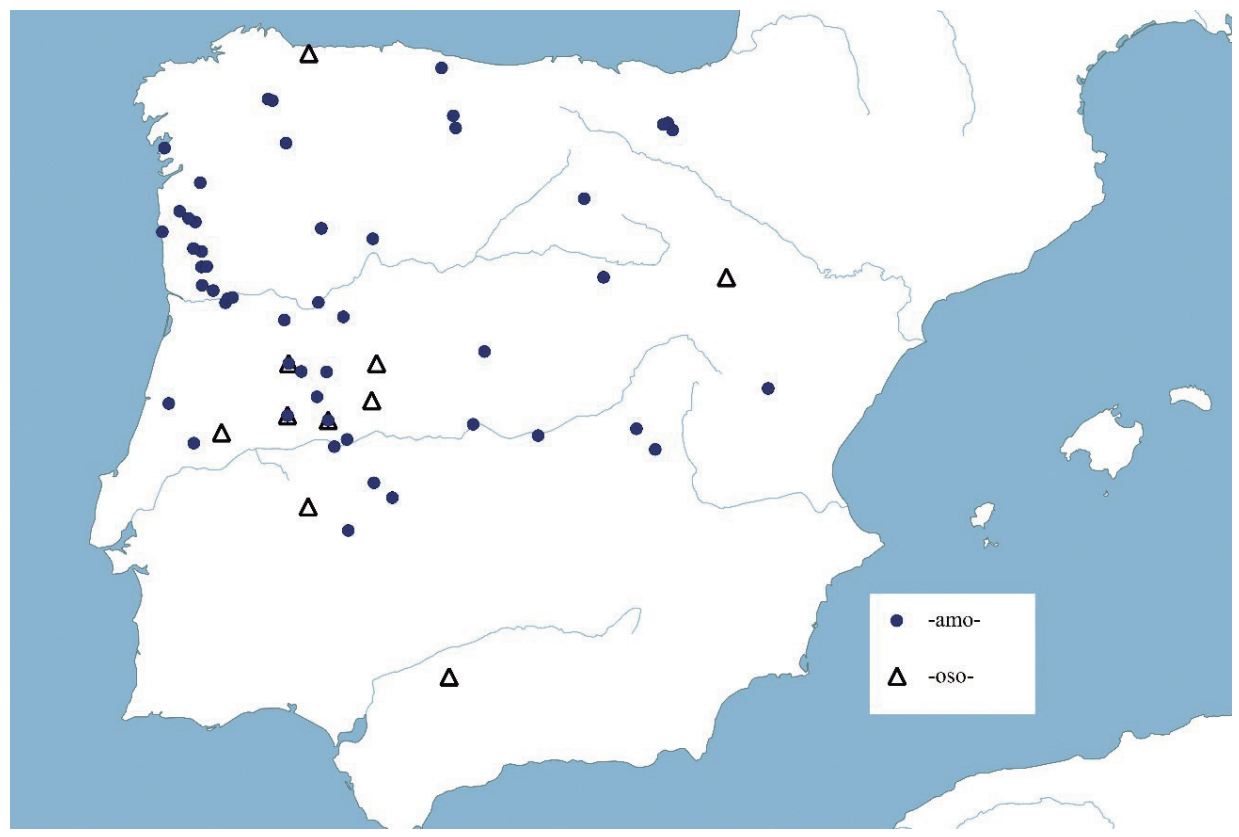


Con el sufijo -amo- podemos encontrar los nombres como: Amparamus, Andamionius, Andamus, Argamonica, Clodameus, Cludamus/Clutamus, Cutamiq(um), Dudamus, Elciamo, Gutamo/Gutamus, Medamus, Melaman(i)us, Melamus, Paramaeco, Paramo, Pentamus, Pintameus, Pintamus, Rotamus, Spantamicus, Toncetamus/Tongetamus, Turaesamicio, Turaesamus, Turamus, Turesamus, Voramos y Vramus. Segisamos no está incluido porque parece ser una formación de superlativo en -is-mimo-.

Y con el formante -OSO- documentamos:

Annosius, Bolos(e)a, Bolosus, Caccossa, Clutosus, Mocosa y Segossoq(um). Las referencias concretas pueden consultarse en Vallejo 2016 o en la Base onomástica del Banco de Datos Hesperia.

\section{Mapa 6. -aiso-}

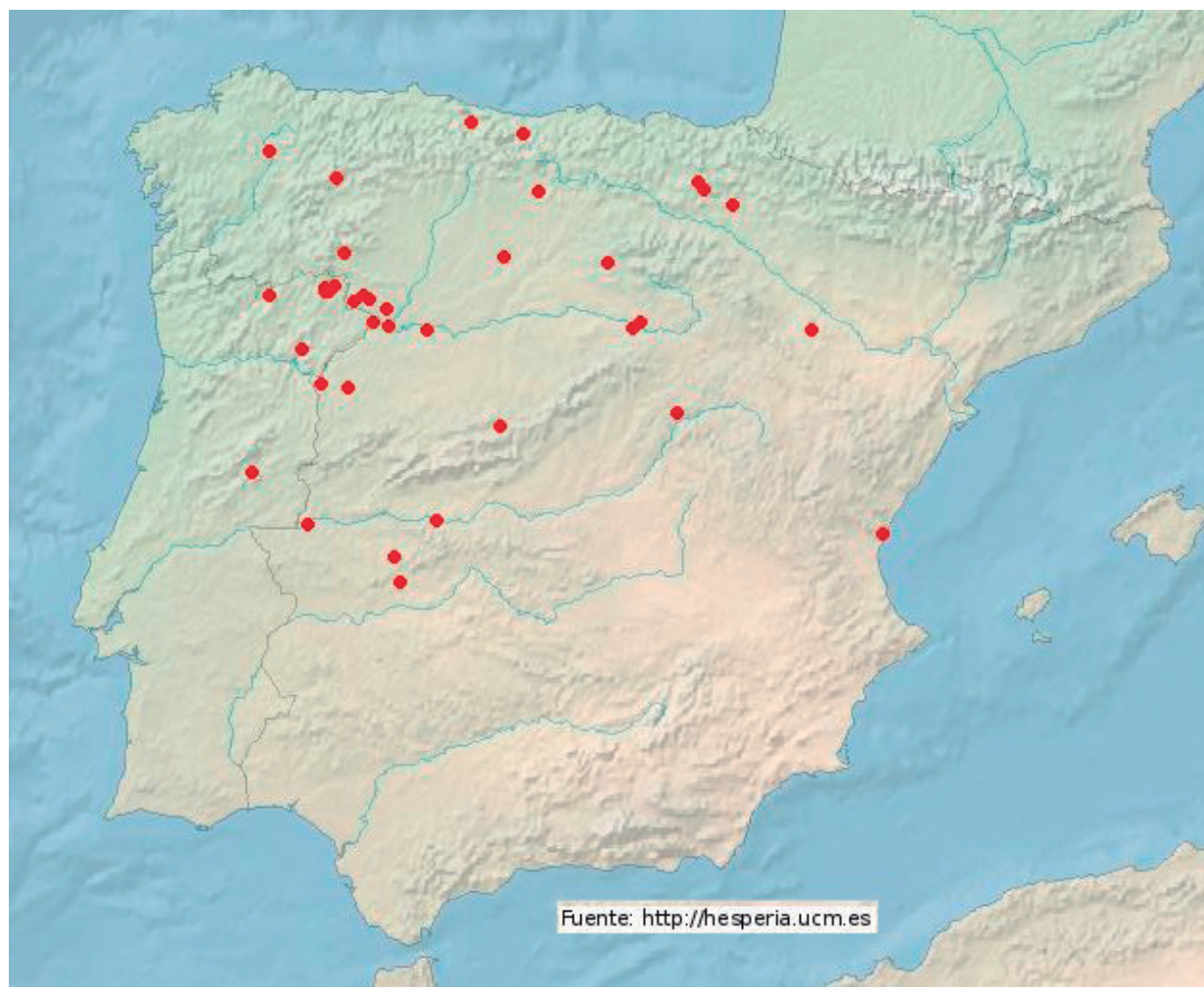


Los nombres con el sufijo -aiso- son los siguientes (cf. Vallejo 2013 y el Banco de Datos Hesperia):

Alaisus, Balaesus/Balaisa, Balesinus, -a, balaisokum, Elaesus, -a/Elesus, Elaisicum, Elaesisca, Elesigainum, Elaisicum, Equaesus/Equesus, Galaesus, Tabaesus, Turaesius, -a, Turaesamus, Turaesamicio, uerzaizokum y Venaesinus.

\section{MAPA 7. -aSO-}

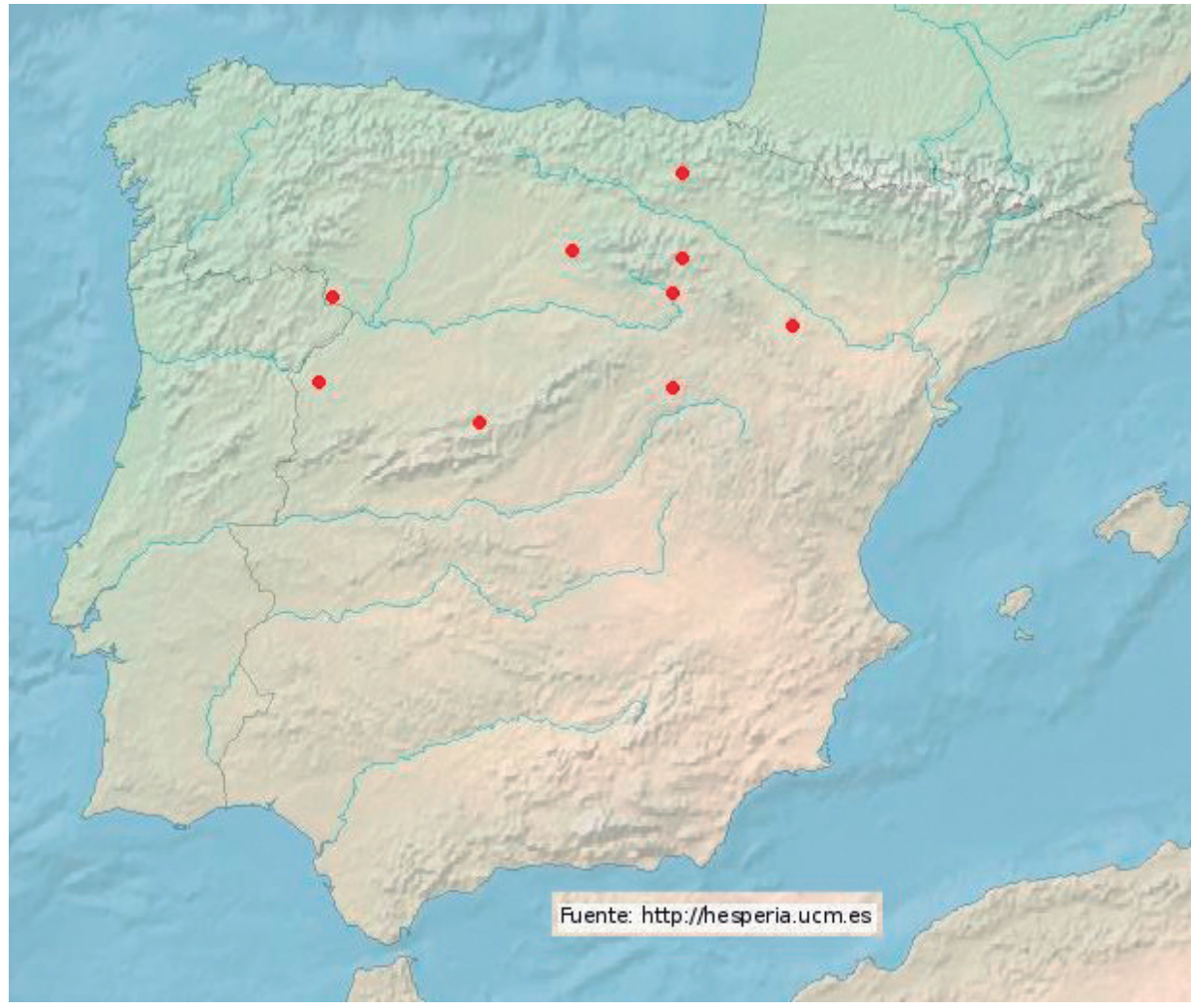

El sufijo -aso- aparece en los nombres:

Ambasia, Attasis, Ciliasa, Elasus, kamasios, Leonasico, Telassicus, Vascasus y Vbasus, cuyas referencias están disponibles en la Base Hesperia. 
Abreviaturas

Hesperia: Banco de Datos Hesperia (http://hesperia.ucm.es). Para la antroponimia, cf. http://hesperia.ucm.es/consulta_hesperia/onomastica/acceso_onomastica.php

IEW: Pokorny, Julius. Indogermanisches etymologisches Wörterbuch. Bern München: Francke Verlag, 1959.

LIV: Rix, Helmut (coord.). Lexikon der indogermanischen Verben (Die Wurzeln und ihre Primärstammbildungen), unter Leitung von Helmut Rix, bearbeitet von Martin Kümmel, Thomas Zehnder, Reiner Lipp, Brigit Schirmer, 2. Auflage, Wiesbaden, 1998.

NIL: Wodtko, Dagmar S., Britta Irslinger, y Carolin Schneider. Nomina im Indogermanischen Lexikon. Heidelberg: Universitätsverlag, 2008.

Bibliografía

Albertos Firmat, M. ${ }^{a}$ Lourdes. La onomástica personal primitiva de Hispania: Tarraconense y Bética. Salamanca, 1966.

Albertos Firmat, M. ${ }^{a}$ Lourdes. "Onomastique personnelle indigène de la Péninsule Ibérique sous la domination romaine». ANRW II, 29. 2 (1983): 853-892.

Bechtel, Friedrich. Historische Personennamen des Griechischen bis zur Kaiserzeit. Halle [Hildesheim, Zürich, New York], 1917 [1982].

Buesa Oliver, Tomás. «Recursos fónicos en la afectividad de los antropónimos». En Actas del I Congreso Internacional de Historia de la Lengua Española (Cáceres 1988), vol. 2, 1613-1640. Madrid: Arco Libros, 1988.

Chambon, Jean-Pierre y Christian Hérilier. "À propos de l'interface onomastique / lexicologie (lexicographie) : Fragments provisoires pour une approche axiomatisée en toponymie». En Actas do XIX Congreso Internacional de Lingüística e Filoloxía Románica (Santiago de Compostela, 1989), vol. IV, 441-451. La Coruña: Fundación 'Pedro Barrié de la Maza', 1993.

Correa, José Antonio. «Identidad, cultura y territorio en la Andalucía prerromana a través de la lengua y la epigrafía». En Identidades, culturas y territorios en la Andalucía prerromana, coordinado por F. Wulff Alonso y M. Álvarez Martí Aguilar, 273-295. Málaga, 2009.

De Hoz, Javier. Historia lingüistica de la península ibérica en la Antigüedad. I: Preliminares y mundo meridional prerromano. Madrid, 2010. 
Evans, D. Ellis. Gaulish Personal Names. A Study of Some Continental Celtic Formations. Oxford University Press, 1967.

Fick, August. Die griechischen Personennamen nach ibrer Bildung erklärt. Göttingen, 1874.

Gorrochategui, Joaquín. «Los Pirineos entre Galia e Hispania: las lenguas». Veleia 12 (1995): 181-234.

Gorrochategui, Joaquín y José M. ${ }^{a}$ Vallejo. "The parts of Hispania without epigraphy». En Palaeohispanic Languages E Epigraphies, editado por A. G. Sinner y J. Velaza, 335-364. Oxford: Oxford University Press, 2019.

Hilka, Alfons. "Beiträge zur Kenntnis der indischen Namengebung. Die altindischen Personennamen». Indische Forschungen 3 (1910): 55-63.

Humboldt, Wilhelm von. Prüfung der Untersuchungen über die Urbewohner Hispaniens vermittelst der baskischen Sprache. Berlin, 1821. Versión española Investigaciones sobre los primitivos habitantes de España con ayuda de la lengua vasca. Trad. de Luis Rivero. Madrid: Ediciones Polifemo, 1990.

Matasović, Ranko. Etymological Dictionary of Proto-Celtic. Leiden - Boston: Brill, 2009.

Meyer, Kuno. "Die Koseformen irischer Personennamen». En "Zur keltischen Wortkunde» I-IX, Sitzungsberichte der königliche preussischen Akademie der Wissenschaften, § 33, 1912-1919.

Nicolaisen, Wilhelm Fritz Hermann. "Onomastic onomastics». En Actes du XVIe Congrès International des Sciences Onomastiques (Québec, Université Laval, 16-22 août 1987), editado por J.-C. Boulanger, 3-14. Québec: Ed. Boulanger - Université Laval, 1990.

Palomar Lapesa, Manuel. La onomástica personal pre-latina de la antigua Lusitania. Estudio lingüístico. Salamanca: CSIC, 1957.

Pokorny, Julius. Reseña a K. H. Schmidt (1957) «Die Komposition in gallischen Personennamen». Kratylos 3 (1958): 171-173.

Pulgram, Ernst. «Indo-European personal names». Language 23 (1947): 189-206.

Pulgram, Ernst. "New evidence on Indo-European personal names». Language 36 (1960): 198-202.

Raybould, Marilynne E. y Patrick Sims-Williams. The Geography of Celtic Personal Names in the Latin Inscriptions of the Roman Empire. Aberystwyth, 2007a.

Raybould, Marilynne E. y Patrick Sims-Williams. A Corpus of Latin Inscriptions of the Roman Empire containing Celtic Personal Names. Aberystwyth, 2007b. 
Raybould, Marilynne E. y Patrick Sims-Williams. Introduction and Supplement to the Corpus of Latin Inscriptions of the Roman Empire containing Celtic Personal Names. Aberystwyth, 2009.

Rubio Alija, José. «Españoles por los caminos del imperio romano. Estudios epigráfico-onomásticos en torno a Reburrus y Reburrinus». Cuadernos de Historia de España 29-30: (1959) 5-124.

Rubio Orecilla, Francisco. «Las formaciones secundarias en - $k o-$ del celtibérico». En Religión, Lengua y Cultura Prerromanas en Hispania, 581591. Salamanca: Ed. Universidad de Salamanca, 2000.

Schmidt, Karl Horst. «Die Komposition in gallischen Personennamen». ZCP 26 (1957): 33-301.

Untermann, Jürgen. Sprachräume und Sprachbewegungen im vorrömischen Hispanien. Wiesbaden, 1961.

Untermann, Jürgen. Elementos de un atlas antroponímico de la Hispania antigua. Madrid, 1965.

Untermann, Jürgen. MLH III = Monumenta Linguarum Hispanicarum, III: Die iberischen Inschriften aus Spanien. Wiesbaden, 1990.

Vallejo, José M. ${ }^{2}$. Antroponimia indígena de la Lusitania romana. VitoriaGasteiz, 2005a.

Vallejo, José M. ${ }^{a}$. "La composición en la antroponimia antigua de la Península Ibérica». Acta Palaeohispanica IX. Actas del IX Coloquio sobre lenguas y culturas paleohispánicas (Barcelona, 20-24 de octubre de 2004) (= Palaeohispanica 5). Zaragoza (2005b): 99-134.

Vallejo, José M. ${ }^{a}$. "La validez de la antroponimia como fuente de estudio de las lenguas antiguas: el caso de la Península Ibérica». Emerita 77 / 1 (2009): 125-145.

Vallejo, José M. ${ }^{a}$. Onomástica paleohispánica. Antroponimia y teonimia: Testimonios epigráficos latinos, celtibéricos y lusitanos, y referencias literarias, Banco de Datos Hesperia de Lenguas Paleohispánicas (BDHESP III, I, 1). Bilbao: Universidad del País Vasco, 2016.

Zimmer, Heinrich. "Keltische Studien, 10. Zur Personennamenbildung im Irischen». Kubns Zeitschrift für vergleichende Sprachforschung 32 (1893): 158-197. 